\title{
Evo-devo como disciplina integradora: la temporalidad de los procesos biológicos como estrategia de análisis ${ }^{*}$ \\ (Evo-Devo as an Integrative Discipline: the temporality of biological processes as a strategy of analysis) \\ Constanza Alexandra RENDÓN y Guillermo FOLGUERA
}

Received: 27.02 .2014

Final version: 02.07.2014

BIBLID [0495-4548 (2014) 29: 81; pp. 395-415]

DOI: $10.1387 /$ theoria.11020

RESUMEN: El objetivo del presente trabajo es indagar la naturaleza integradora de la biología evolutiva del desarrollo (evo-devo). En particular analizamos las características temporales de los procesos estudiados por diferentes programas de investigación de evo-devo y las comparamos con aquellas propias de los procesos macroevolutivos, microevolutivos y del desarrollo de los organismos. Encontramos que en los principales programas de investigación de evo-devo se recuperan principalmente características propias de los fenómenos macroevolutivos (planteándose cierta continuidad entre tales fenómenos y los cambios genéticomoleculares y organísmicos), mientras que en la sub-área de eco-evo-devo (biología evolutiva ecológica del desarrollo) se recuperan principalmente características propias de la microevolución.

Palabras clave: características temporales; procesos biológicos; evolución; desarrollo ontogenético; síntesis moderna; eco-evo-devo.

ABSTRACT: The aim of this study is to investigate the integrative nature of evolutionary developmental biology (evodevo). We particularly analyzed the temporal characteristics of the processes studied by different research programs of evo-devo and we compared them with those of macroevolution, microevolution and developmental processes of organisms. We found that in the major research programs of evo-devo, macroevolutionary phenomena characteristics are mainly recovered (assuming some continuity between such phenomena and molecular-genetic and organismic changes), while in the sub-area of eco-evo-devo (ecological evolutionary developmental biology) microevolutionary characteristics are mainly recovered.

Keywords: temporal characteristics; biological processes; evolution; development; modern synthesis; eco-evo-devo.

\section{La diversidad de los procesos biológicos y su estudio desde una biología fragmentada}

La conceptualización de la vida asociada a la idea de cambio y devenir se consolidó hacia el siglo XIX. Los procesos propios de lo vivo fueron constituyéndose así en temas de estudio de la naciente biología. Entre ellos se destacó tanto la indagación de los denominados procesos ontogenéticos como la de los evolutivos. Sin embargo, la integración de ambos tipos de procesos ha distado de ser sencilla. Desde el siglo XIX pueden reconocerse importantes intentos de abordar la evolución y la ontogenia en forma integrada, tales como las propuestas de Ernst Haeckel y August Weismann (Gould 2002; Laubichler y Maienschein 2007; Rendón y Folguera 2011). Sin embargo, a partir de la consolidación de la denominada síntesis moderna (SM) en la década de 1930, el estudio de cada uno de estos procesos comenzó a separarse. La SM ubicó en

\footnotetext{
* Agradecemos a la Dra. Olimpia Lombardi y a los revisores anónimos por sus valiosas correcciones y comentarios, los cuales nos han permitido enriquecer y mejorar este trabajo.
} 
un lugar central a la genética mendeliana y a la genética de poblaciones como subdisciplinas que explican las causas del cambio evolutivo, excluyendo a la embriología y a otras áreas de conocimiento (tales como la morfología) del estudio de la evolución (Fox Keller 2000; Amundson 2005; Love 2006). ${ }^{1}$ Si bien desde su origen la SM fue objeto de diversos cuestionamientos, fue hacia la década de 1970 cuando cobraron gran notoriedad diversas posturas que criticaron aquella asignación de roles subdisciplinares. Uno de los principales cuestionamientos remitió al tipo de relación asumida entre la denominada microevolución (evolución de las poblaciones) y la macroevolución (evolución de los linajes superiores) (Gould y Eldredge 1977; Gould y Lewontin 1979). Paralelamente, también cobraron fuerza las posturas que reivindicaban el estudio de los procesos ontogenéticos y de sus vínculos con los procesos evolutivos (Gould 1977; Alberch et al. 1979; Hall 1984; Raff y Wray 1989). Finalmente, durante la década de 1990, se consolidó el surgimiento de una nueva área de estudio: la biología evolutiva del desarrollo (evolutionary developmental biology o evo-devo). La evo-devo propuso como uno de sus principales objetivos integrar el estudio de los procesos micro y macroevolutivos con los propios del desarrollo ontogenético de los organismos (Amundson 2005; Laubichler y Maienschein 2007; Müller 2008).

Sin embargo, la empresa integradora de evo-devo no ha estado exenta de críticas, las cuales han surgido desde diversos campos de investigación. Entre los principales aspectos cuestionados pueden señalarse: la prioridad que en gran parte de las investigaciones de evo-devo se ha dado al estudio del nivel genético-molecular (Hendrikse et al. 2007; Rendón et al. 2012; Rendón 2013), el papel que el estudio del desarrollo ontogenético ha tenido en esta integración (Robert 2002) y la exclusión de otras subdisciplinas que han abordado otros aspectos de los procesos evolutivos (Love 2006). Asimismo, han sido señaladas dificultades conceptuales involucradas en la integración de áreas de estudio que presentan metodologías, enfoques y estándares explicativos diferentes (Amundson 2005; Laubichler y Maienschein 2007).

No obstante uno de los elementos conceptuales que no ha recibido suficiente atención en los análisis acerca de la evo-devo es el de los aspectos temporales propios de los procesos indagados en este campo de estudio (cabe mencionar aquí los trabajos de Cracraft (2005) y Rasskin-Gutman y Esteve-Altava (2009) que tratan tangencialmente este tema). Desde nuestra perspectiva el análisis de la temporalidad de los procesos abordados desde la evo-devo resulta de sumo interés e importancia debido a su propósito de integrar el estudio de procesos del desarrollo ontogenético con los micro y macroevolutivos, los cuales presentan características temporales marcadamente diferentes.

A partir de lo señalado, el objetivo del presente trabajo es reconocer y analizar las características relativas a la temporalidad de los procesos propios del área de evo-devo, y compararlas con aquellas presentes en otros enfoques de la evolución y del desarro-

\footnotetext{
1 Se han planteado diversos motivos para explicar la exclusión de la embriología de la SM. La aceptación generalizada de la propuesta de Weismann de separación entre línea somática y línea germinal ha influido en el establecimiento y la justificación de tal exclusión, así como otras dicotomías conceptuales defendidas desde la SM (ver Amundson 2005).
} 
llo ontogenético de los organismos. De esta manera pretendemos profundizar el conocimiento acerca de la propia naturaleza integradora de la evo-devo. Para ello el trabajo está estructurado del siguiente modo: en el próximo apartado (sección 2) proponemos una taxonomía basada en cuatro características de los procesos biológicos asociadas a la temporalidad (tipo de trayectoria, reversibilidad, tasa de cambio y duración). En la tercera sección, analizamos las características temporales propias de los procesos evolutivos y del desarrollo ontogenético de los organismos, en particular: la conceptualización de los cambios microevolutivos que se ha dado desde la SM (sección 3.1), el análisis de los procesos macroevolutivos en la extensión de la SM durante las décadas de 1970 y 1980 (sección 3.2), y el estudio de los procesos ontogenéticos desde la perspectiva de la actual biología del desarrollo (sección 3.3). En la sección 4 indagamos las características de los procesos abordados por diferentes programas de evodevo, incluyendo como una sub-área dentro de ella a la biología evolutiva ecológica del desarrollo (ecological evolutionary developmental biology o eco-evo-devo). Finalmente en la sección 5 presentamos las conclusiones acerca de las características temporales de los procesos estudiados en evo-devo y sus implicaciones respecto de la integración de diferentes campos de estudio en esta propuesta de extensión de la SM.

\section{Taxonomía de características asociadas a la temporalidad de los procesos biológicos}

En el presente trabajo entenderemos por proceso a una sucesión de estados a la que subyace un mecanismo. Con el fin de caracterizar los procesos biológicos procuramos identificar a las entidades involucradas, las actividades o funciones de esas entidades y su organización. Tal como adelantamos en la sección anterior, utilizaremos una taxonomía que hemos elaborado en el presente trabajo para el análisis de cuatro características temporales de los procesos: tipo de trayectoria, reversibilidad, tasa de cambio y duración. A continuación definiremos brevemente cada una de ellas.

\section{1) Tipo de trayectoria}

Esta categoría describe el orden presente en la sucesión de los estados que conforman los procesos. En el presente trabajo distinguimos tres tipos de trayectoria:

a) Trayectoria lineal: denominaremos trayectoria lineal a aquella en la cual se sucedan estados diferentes a través del tiempo de manera tal que el proceso presente dirección y sentido. La identificación de la característica o variable del proceso que le confiere tal direccionalidad dependerá de cada proceso en particular. El crecimiento de un organismo es un ejemplo de proceso lineal al cual el aumento de ciertas variables morfológicas (por ejemplo, la estatura) confiere dirección y sentido.

b) Trayectoria cíclica o recurrente: nos referiremos a procesos cíclicos cuando se presente alternancia entre al menos dos estados reiteradamente, de la siguiente manera:

$$
E_{1}-E_{2}-E_{1}-E_{2}-E_{1}-E_{2} \ldots
$$

donde $\mathrm{E}_{1}$ y $\mathrm{E}_{2}$ representan diferentes estados del proceso. 
Los ritmos circadianos presentes en los seres vivos (tales como la alternancia entre los estados de sueño y vigilia en los animales) constituyen ejemplos de procesos cíclicos.

c) Trayectoria helicoidal o cuasi-recurrente: esta trayectoria surge a partir de una característica propia de ciertos procesos biológicos, la recursividad (Morin 1983). Esta propiedad se refiere a que el estado final del proceso es necesario y suficiente para la regeneración del propio proceso. En los procesos biológicos suele encontrarse recursividad no estricta, dado que no se repiten estados exactamente iguales a los anteriores, sino similares morfológica y/o funcionalmente (y generados a partir de los productos o efectos de estados previos). La trayectoria de este recorrido puede visualizarse entonces como un bucle o hélice, la cual puede representarse como:

$$
\mathrm{E}_{1}-\mathrm{E}_{2}-\mathrm{E}_{3} \ldots \mathrm{E} n-\mathrm{E}_{1}^{\prime}-\mathrm{E}_{2}^{\prime} \ldots,
$$

donde $\mathrm{E} n$ es condición necesaria y suficiente para generar $\mathrm{E}_{1}{ }^{\prime}, \mathrm{y}$ los $\mathrm{E} n^{\prime}$ representan estados similares a los $\mathrm{E} n$ correspondientes.

Un ejemplo de proceso helicoidal es la re-generación del ciclo de vida de los organismos en cada nueva generación. Determinados estados generados en el ciclo de vida de la generación parental constituyen condiciones necesarias y suficientes para el re-comenzar del ciclo en una nueva generación, en la cual a su vez se desarrollarán estados similares (mas no idénticos) a los del ciclo que les dio origen.

\section{2) Reversibilidad}

Diremos que un proceso es reversible en el caso en que la sucesión de estados presentes admita en su secuencia la regresión a un estado anterior. Un caso de reversión podría presentarse de la siguiente manera:

$$
E_{1}-E_{2}-E_{3}-E_{2}-E_{3}-E_{4} \ldots E n
$$

\section{3) Tasa de cambio}

Emplearemos esta categoría para referirnos en forma relativa a la variación de velocidad en la transición entre diferentes estados. Utilizaremos la denominación de procesos uniformes para referirnos a aquellos que presenten una tasa de cambio constante; en caso contrario, denominaremos a la tasa de cambio como variable.

\section{4) Duración}

Por último, analizaremos la duración de los procesos, categoría que se refiere a la extensión del intervalo de tiempo comprendido entre el comienzo del estado inicial y la culminación del estado final del proceso. Realizaremos mayoritariamente comparaciones cualitativas entre la duración de los diferentes tipos de procesos.

En la sección siguiente utilizaremos esta taxonomía para caracterizar los aspectos temporales de los procesos evolutivos y ontogenéticos. 


\section{De la sintesis moderna a la evo-devo: fragmentación e integración}

\subsection{Procesos microevolutivos: reversibilidad y uniformidad en la evolución de las poblaciones}

Como adelantamos, entre las décadas de 1940 y 1960, la SM identificó a la evolución con el ámbito microevolutivo. En este sentido los procesos evolutivos fueron entendidos como aquellos que generan cambios en las frecuencias génicas de las poblaciones (Ridley 2004). ${ }^{2}$ Para explicar dichos cambios fueron propuestos diferentes mecanismos: selección natural (como principal mecanismo evolutivo), migración, mutación y deriva génica. A su vez, un elemento fundamental en este enfoque de la evolución ha sido la novedosa metodología utilizada: los cambios de frecuencias génicas fueron estudiados en poblaciones experimentales, imitando así los procesos microevolutivos en el laboratorio. Además los modelos matemáticos propios de la genética de poblaciones ocuparon un lugar central en este enfoque. Al mismo tiempo, la macroevolución fue considerada como un continuum de los cambios poblacionales, y su estudio no fue incorporado como parte del núcleo teórico central de la SM. El rol de la paleontología en el estudio de la evolución quedó así reducido al de mero presentador fenoménico.

Para comenzar con el análisis de las características temporales de los procesos microevolutivos, nos centraremos en aquellos que ocurren por acción de selección natural direccional, una de las principales formas de evolución modeladas desde la SM. Consideraremos un escenario simple: la evolución de un gen que presenta dos variantes alélicas cuyas frecuencias poblacionales son diferentes (por ejemplo, 0.1 y 0.9), y selección direccional que favorece a la variante de menor frecuencia inicial. Los modelos de genética de poblaciones postulan que las frecuencias génicas se modificarán a través del tiempo de forma tal que la aptitud media de la población aumente hasta alcanzar el máximo valor posible dadas las condiciones específicas en cada situación (en este caso, se predice el aumento de frecuencia poblacional de la variante de menor frecuencia inicial). Este principio se evidencia tanto en los modelos de selección direccional como en otros modelos selectivos. Así, la dirección y sentido de estos procesos microevolutivos están dados por la optimización o maximización de la aptitud media poblacional. Además, este principio 'direccionador' de los procesos selectivos ha sido descrito mediante 'topografías adaptativas', las cuales representan las posibles combinaciones de frecuencias alélicas de todos los genes en una población. Estas superficies contienen un 'pico adaptativo' correspondiente a la máxima aptitud media posible en esa topografía. Según el principio de maximización de esa variable bajo la acción de la selección natural, se espera que las poblaciones alcancen ese pico adaptativo. ${ }^{3}$ Veamos entonces qué características temporales presentan estos procesos.

2 Cabe mencionar que desde el surgimiento de la SM existieron diversas posturas en su seno respecto de esta concepción de los procesos evolutivos. Si bien tal noción fue rechazada por algunos artífices de la SM, como Ernst Mayr, también ha sido defendida por otros, como Theodosius Dobzhansky (Amundson 2005). Más allá de la diversidad de posturas dentro de la SM y de los cambios que sufrió este marco teórico a lo largo del siglo XX, la citada conceptualización de cambio evolutivo ha sido la sostenida en la genética de poblaciones, subdisciplina central en la SM.

${ }^{3}$ Si bien existieron propuestas que presentan características diferentes a esta direccionalidad de los procesos microevolutivos (tales como el modelo de 'equilibrios cambiantes' de Sewall Wright), el modelo descrito ha sido el mayormente aceptado y utilizado por los genetistas de poblaciones. 
Con respecto al tipo de trayectoria, las propuestas canónicas de la SM consideran a estos procesos como lineales, en los cuales la dirección y sentido estarían dados por el principio de aumento de la aptitud media poblacional. A su vez, en relación con la segunda categoría, encontramos que el tipo de proceso analizado admite la posibilidad de reversiones a estados de frecuencias poblacionales anteriores. Un ejemplo clásico de reversibilidad (aunque también discutido, ver por ejemplo Ridley 2004) es el de las poblaciones naturales de las polillas Biston betularia. La frecuencia de los morfos claro y oscuro de los organismos de esta especie se ha modificado a lo largo del siglo XX en respuesta a los cambios en los niveles de polución ambiental, predominando claramente uno de los morfos al aumentar o disminuir la polución. Así, los procesos microevolutivos modelados desde la genética de poblaciones presentan esta dinámica flexible con respecto a la posibilidad de reversión a estados de frecuencias génicas anteriores, debido a los cambios en las presiones selectivas.

Por otra parte, en relación con la tasa de cambio de los procesos microevolutivos, encontramos que en la mayor parte de los modelos de la genética de poblaciones se presenta como uniforme. Esto es consecuencia de que los parámetros de los cuales depende la tasa de cambio de las frecuencias génicas (principalmente, el coeficiente de selección) son considerados constantes en estos modelos. ${ }^{4}$ Expresado en forma cualitativa, el principio de gradualidad de los cambios evolutivos ha sido una de las principales premisas defendidas por los autores de la SM (en expresa oposición a otras posturas acerca de la evolución): dado que las poblaciones se encuentran medianamente adaptadas, los cambios abruptos las alejarían indefectiblemente del pico de la correspondiente topografía adaptativa. Por ello, sólo los pequeños cambios permitirían que las poblaciones se acerquen, lenta y gradualmente, al pico adaptativo correspondiente y subsistan.

Por último, los procesos microevolutivos se desarrollarían en una escala temporal relativamente breve en términos evolutivos, es decir, en pocas generaciones (en concordancia con la metodología experimental utilizada para su estudio). A su vez, se asume que los cambios macroevolutivos surgirían debido a la acumulación de pequeños cambios a lo largo de un intervalo temporal de mayor duración (Mayr 1942; Simpson 1953). El cuestionamiento a este principio será una de las principales críticas hacia la SM, como desarrollaremos en la sección siguiente.

\subsection{La extensión macroevolutiva: discontinuidades e irreversibilidad en la historia de la vida}

Como adelantamos, hacia la década de 1970 cobró gran relevancia una corriente de críticos a la SM quienes cuestionaron la explicación de los fenómenos macroevolutivos (tales como el origen de nuevas especies o la extinción de grandes taxa) a través de mecanismos microevolutivos. ${ }^{5}$ Desde estas perspectivas se planteó que era necesario

4 Si bien en principio es posible que los valores de tales parámetros cambien de una generación a la siguiente, esto no es lo usualmente planteado en los modelos generales de la genética de poblaciones.

5 Este cuestionamiento puede reconocerse anteriormente en diversas propuestas tales como las de Richard Goldschmidt y Rupert Riedl, entre otros (Gould 2002; Laubichler y Maienschein 2007). 
reconocer procesos específicos que generen cambios propios de los taxa superiores. En este sentido se resaltó el rol del análisis morfométrico, tanto de fósiles como de especies actuales, reivindicándose los enfoques propios de la paleontología y la morfología (Eldredge y Gould 1972; Gould y Eldredge 1977; Alberch et al. 1979; Wake et al. 1983; Seilacher 1984). Además se propusieron nuevos modelos teóricos para abordar el estudio de los cambios macroevolutivos y novedosas hipótesis referentes a la naturaleza de dichos cambios. Una de las principales propuestas al respecto apunta justamente a las características temporales de los procesos macroevolutivos: la 'teoría de los equilibrios puntuados o discontinuos' de Eldredge y Gould (1972).

La diferencia más evidente entre dicha propuesta y las consideraciones de la SM corresponde a la tasa de cambio no uniforme de los procesos macroevolutivos. Brevemente, el modelo de equilibrios puntuados propone que las especies transcurren la mayor parte de su historia sin sufrir grandes cambios (los denominados períodos de estasis). En contraposición, los cambios macroevolutivos ocurrirían en períodos de tiempo relativamente breves (miles de años). Así, al ampliar la escala de tiempo abordada respecto de las propuestas de la SM, la interpretación del registro fósil indicaría que los procesos macroevolutivos no ocurren uniformemente a través del tiempo ni en escalas temporales compatibles con su imitación en el laboratorio.

Por otra parte, con respecto a las restantes categorías de nuestra taxonomía temporal, encontramos que varias de las características propias de los procesos macroevolutivos se relacionan con la consideración de que éstos constituyen procesos históricos ${ }^{6}$. Por lo tanto, además de las propuestas de modelos e hipótesis generales acerca del modo y tempo de tales cambios, se propone la reconstrucción de la historia evolutiva de los organismos a través de la elaboración de narraciones históricas. En este sentido Gould señala que:

[La historia es una] irreversible secuencia de eventos irrepetibles. Cada momento ocupa su propia posición distintiva en una serie temporal, y todos los momentos, considerados en su adecuada secuencia, cuentan una historia de eventos unidos moviéndose en una dirección. $(1987,11)$

Así, en estas propuestas se destaca la linealidad e irreversibilidad de los procesos macroevolutivos. El azar y la contingencia juegan aquí un rol fundamental participando en la generación de acontecimientos únicos en la historia de la vida (Gould 1987; 2002).

Finalmente, la duración de los procesos macroevolutivos sería mayor que la de los microevolutivos. Cabe aclarar que si bien los procesos de especiación ocurren en intervalos de tiempo cortos respecto de los periodos de estasis, aquellos suelen abarcar intervalos de mayor duración que la evolución de las poblaciones en el marco de la SM. Esta característica, sumada a la consideración de la naturaleza histórica de los procesos macroevolutivos, posiblemente haya influido en que no se desarrollaran propuestas metodológicas que implicaran su imitación en el laboratorio.

${ }^{6}$ En estas propuestas se recupera la noción de la historia como una secuencia de fenómenos irrepetibles debido a la "improbabilidad estadística de que un número incalculable de configuraciones independientes que anteceden y componen cualquier evento histórico ocurran dos veces" (Gould 1970, 208). La naturaleza histórica e irreversible de los procesos macroevolutivos ha sido extensamente discutida por Gould (1970; 1987; 2002), entre otros autores. 


\subsection{Los procesos ontogenéticos: la irreversibilidad de la construcción recursiva de los organismos}

Como adelantamos, a partir de la consolidación de la SM, el estudio de los procesos ontogenéticos fue excluido del estudio de la evolución. Posteriormente, hacia la década de 1960, la embriología comenzó a sufrir importantes modificaciones (principalmente mediante la incorporación de metodologías de la biología molecular y la consolidación de un enfoque cada vez más genético), adquiriendo la denominación de biología del desarrollo (Fox Keller 2000). Esta subdisciplina aborda el estudio de los procesos de morfogénesis (formación de órganos y tejidos) y de los diversos cambios que sufren los organismos en su desarrollo, los cuales involucran crecimiento y diferenciación de estructuras a través del ciclo de vida. Estos procesos se relacionan además con cambios en la fisiología y en la expresión genética, por lo cual el estudio de la sucesión de estados transitorios del desarrollo involucra el análisis de múltiples niveles biológicos (genético-molecular, celular, tisular, organísmico).

Antes de comenzar con el análisis de las características temporales de estos procesos resulta pertinente realizar una distinción entre dos tipos de enfoques presentes en la biología del desarrollo: un conjunto de trabajos centrados en los cambios estructurales de órganos y tejidos, continuador de la tradición anatómica propia de la embriología desde sus comienzos (por ejemplo, Kampermann et al. 1997; Denver 1998; Krain y Denver 2004), y otro tipo de estudios centrados en los cambios en la expresión génica y los procesos moleculares relacionados con la diferenciación de tejidos y órganos (tales como Cohn y Tickle 1999; Zúñiga et al. 1999; Basch et al. 2006). Dado que estos dos enfoques presentan características temporales diferentes, analizaremos a continuación cada uno de ellos por separado.

En primer lugar, la tradición anatómica se ha centrado en la descripción de la aparición y modificación de estructuras, tejidos y órganos en las diferentes etapas del desarrollo de diversas especies. Por ejemplo, los estudios del ciclo de vida de los anuros y de las etapas del desarrollo embrionario de las aves constituyen modelos clásicos de la subdisciplina hasta la actualidad. Cabe señalar que en los trabajos de investigación actuales los estudios anatómicos se encuentran generalmente acompañados del estudio de los genes y/o moléculas relacionados con los cambios morfológicos. Sin embargo, pueden encontrarse algunos trabajos que, si bien analizan también factores genéticos y moleculares, tienen como objetivo principal el estudio de cambios estructurales y fisiológicos en diferentes etapas del desarrollo o ante diferentes perturbaciones (ver por ejemplo Kempermann et al. 1997; Denver 1998; Krain y Denver 2004).

Comenzando con el análisis del tipo de trayectoria temporal involucrada en el ciclo de vida cabe señalar que la misma denominación de 'ciclo' remite a la repetición, generación tras generación, de los mismos estadios durante el desarrollo de los organismos de cada especie. Sin embargo, al analizar cómo se estudian estos ciclos, encontramos que el objeto de investigación es algún/os aspecto/s de la formación de estructuras durante alguna/s etapa/s del desarrollo (tal como en Denver 1998; Krain y Denver 2004), sin incluir el estudio de lo que ocurre en las generaciones subsiguientes. De esta manera, al omitir un enfoque transgeneracional, se excluye el aspecto cíclico de estos procesos. Así, el desarrollo es abordado como una secuencia lineal e irreversible, que 
se repite en la vida de cada organismo. Estas características se relacionan con los mecanismos celulares y tisulares involucrados en los procesos morfogenéticos:

El hecho de que muchos eventos dependan de la inducción, o las interacciones entre células y tejidos, o de las interacciones complejas dentro de varias cascadas génicas significa que existe una direccionalidad fundamental en el desarrollo. (Smith 2003, 619)

Así, el modo en que se expresa la linealidad propia de estos procesos es el aumento progresivo de la complejidad a través del desarrollo. En términos generales este incremento de complejidad se relaciona con un aumento de la heterogeneidad estructural y funcional, y la emergencia de entidades, propiedades e interacciones propias de nuevos niveles de organización a lo largo del desarrollo. La complejidad de tales interacciones implica también la irreversibilidad involucrada generalmente en estos procesos. Sin embargo, cabe mencionar algunos casos excepcionales en los cuales es posible revertir, en condiciones particulares, ciertos procesos del desarrollo. Tales reversiones pueden apreciarse en los experimentos de desdiferenciación de núcleos celulares (por ejemplo, en los experimentos de clonación) o de regeneración de ciertos tejidos.

Por otra parte, con respecto a la tasa de cambio, encontramos que, en términos generales, se asume que es variable a lo largo del desarrollo. Un caso extremo de tal variabilidad es el arrestamiento completo del desarrollo tal como sucede por ejemplo en las estructuras de resistencia de las esporas de algunas bacterias y hongos, las cuales pueden permanecer largos períodos de tiempo en estado de latencia y reactivar su desarrollo ante el estímulo ambiental adecuado.

Por último, con respecto a la duración de estos procesos, el estudio de los ciclos de vida se ha centrado en algún/os estadio/s del desarrollo o, a lo sumo en procesos que ocurren a lo largo de una generación, sin traspasar dicha duración, tal como mencionamos.

Pasando al enfoque que estudia específicamente la expresión genética y los procesos moleculares involucrados en el desarrollo (por ejemplo Cohn y Tickle 1999; Basch et al. 2006) encontramos que gran parte de estos trabajos se centran en las señales moleculares que actúan como inductores de la diferenciación de células y tejidos durante la morfogénesis. Así, el estudio del desarrollo de muchas estructuras (extremidades, tubo neural, entre otras) ha incluido la descripción de las señales intercambiadas recíprocamente por diferentes grupos celulares: al recibir una señal, las células receptoras emiten nuevas señales a las emisoras, las cuales son imprescindibles para que continúe la diferenciación y formación de tejidos, repitiéndose estos 'diálogos celulares' a lo largo de todo el proceso. Un ejemplo en el cual se ha estudiado detalladamente la dinámica involucrada en estos intercambios de señales es el desarrollo de las extremidades de los tetrápodos (Zúñiga et al. 1999). De esta manera la recursividad involucrada en tales diálogos permite caracterizar como helicoidal la trayectoria de estos procesos ya que cada estado concluye con la producción de una señal que genera el pasaje al estado siguiente y retroalimenta el avance del propio proceso.

Con respecto a las restantes categorías temporales encontramos que si bien estos trabajos se centran en la continuidad de los procesos de desarrollo (ya que las inducciones recíprocas generan el avance continuo de los mismos) no se especifica gene- 
ralmente si la tasa de cambio es variable o uniforme. Además, como mencionamos, el aumento del grado de diferenciación y especialización a lo largo de estos procesos implica, generalmente, su irreversibilidad (con las notables excepciones ya mencionadas, en las cuales ciertos procesos genético-moleculares pueden revertirse). Por último, la duración de los mismos abarcaría la propia de algún/os estadio/s del desarrollo, siendo en general menor a la presentada en el enfoque anatómico.

En resumen, la biología del desarrollo ha abordado estos procesos concibiéndolos como una repetición de secuencias en cada generación, cuyos cambios más allá de esa duración quedan fuera del objeto de estudio de la subdisciplina. En contraposición, las relaciones e interacciones entre los procesos del desarrollo y los procesos evolutivos (transgeneracionales) constituyen el objeto de estudio del campo que analizaremos a continuación: la evo-devo.

\section{Evo-devo y la promesa de integración}

Antes de comenzar con al análisis de los procesos estudiados desde evo-devo es necesario destacar la gran diversidad de programas de investigación y propuestas teóricas que han surgido en este campo de estudio (Robert 2002; Laubichler y Maienschein 2007; Müller 2008; Pigliucci y Müller 2010). Basándonos en la clasificación utilizada por Müller (2008) hemos seleccionado para este análisis algunos de los programas de investigación de evo-devo más representados actualmente: la genética evolutiva del desarrollo, la embriología y morfología comparadas, y la sub-área denominada ecoevo-devo (biología evolutiva ecológica del desarrollo).

\subsection{Genética evolutiva del desarrollo}

La genética evolutiva del desarrollo constituye sin duda el programa de evo-devo más representado en la actualidad. Este programa se centra en el estudio de los genes que controlan el desarrollo y de las redes de regulación génica asociadas a la evolución de la forma orgánica (Müller 2008). Específicamente, los procesos abordados son aquellos involucrados en los cambios genético-moleculares (en la secuencia de genes y la función de sus productos en las redes de regulación) vinculados con transformaciones macroevolutivas (principalmente las denominadas innovaciones evolutivas) (Cohn y Tickle 1999; Ronshaugen et al. 2002; Lee et al. 2003; Pick y Heffer 2012). Estos procesos son estudiados comparando la expresión y función de esos genes en el desarrollo de organismos de diversas especies. A partir de estas comparaciones se concluye acerca de los cambios genéticos involucrados en la evolución de las diferencias fenotípicas entre especies o grupos taxonómicos de orden superior. Un caso de estudio paradigmático de este programa es el de los genes hox. Para analizar las características temporales propias de este enfoque utilizaremos el ejemplo de los cambios en los genes hox Ubx y AbdA asociados al desarrollo de patas en crustáceos e insectos (descrito en el trabajo clásico de Ronshaugen et al. 2002). Siguiendo la metodología experimental típica de los trabajos de la genética evolutiva del desarrollo se estableció que los cambios en la secuencia de estos genes en la rama evolutiva que dio origen a los insectos a partir de un antepasado común con los 
crustáceos determinaron su cambio de función: estos genes reprimen la formación de patas en el abdomen de los insectos, no así en el grupo de los crustáceos, los cuales conservan el plan corporal del ancestro común a ambos grupos (con patas en todo el tronco). Así, los cambios en los genes regulatorios del desarrollo contribuyen a explicar los cambios macroevolutivos en la morfología de grandes taxa.

Comenzando con el análisis de las características temporales encontramos en estos procesos un tipo de trayectoria lineal, ya que se busca reconstruir la secuencia de cambios genético-moleculares en la historia de los linajes. De esta manera se recupera en este enfoque la linealidad asociada al carácter histórico de los procesos macroevolutivos. Sin embargo, surge aquí la necesidad de desdoblar nuestro análisis de los procesos estudiados en este programa de investigación dado que encontramos un tipo de trayectoria temporal diferente al centrarnos en los aspectos ontogenéticos involucrados: como los modelos propuestos se basan justamente en el estudio de los procesos genético-moleculares de regulación del desarrollo, se incorpora también la recursividad propia de tales procesos. Por lo tanto, puesto que encontramos que ambos aspectos (evolutivos y ontogenéticos) se encuentran efectivamente presentes en los procesos estudiados desde la evo-devo, distinguiremos para el análisis de las características temporales la dimensión evolutiva de los mismos de su caracterización como procesos del desarrollo. Con respecto a este último aspecto, hallamos que en el programa de la genética evolutiva del desarrollo se asumen las mismas características temporales descritas para los procesos ontogenéticos en la sección 3.3.

Continuando con el análisis de la dimensión evolutiva de los procesos abordados en este programa de investigación, encontramos aquí la idea de que es posible la reversión, de alguna manera, de los cambios macroevolutivos. Esta noción se encuentra implícita en la metodología utilizada ya que se asume que a través de los experimentos que muestran los efectos de las mutaciones en genes regulatorios se imitan (al menos parcialmente) los cambios ocurridos a lo largo de la historia evolutiva de los organismos (Ronshaugen et al. 2002). En este sentido, algunos autores han advertido acerca del alcance 'real' de las conclusiones que presenta el programa de la genética evolutiva del desarrollo, así como de la necesidad de profundizar las indagaciones para respaldar dichas reconstrucciones (Müller 2008).

Por otra parte, en este programa parece asumirse la variabilidad de la tasa de cambio propia de los procesos macroevolutivos. La asociación directa de cambios macroevolutivos con cambios genéticos es compatible con la no uniformidad temporal de aquellos, presentada en la extensión macroevolutiva.

Por último, con respecto a la duración de los procesos estudiados, la genética evolutiva del desarrollo realiza afirmaciones acerca de cambios macroevolutivos a través de una metodología que permitiría imitarlos experimentalmente en tiempos cortos. En este sentido autores como Gould vieron en los comienzos de este programa de investigación la promisoria posibilidad de abordar eventos históricos macroevolutivos a través de una nueva metodología experimental, diferente a la propuesta por los enfoques paleontológicos y morfológicos (Gould 1977; 2002). 


\subsection{Embriología y morfología comparadas}

El programa de la embriología y la morfología comparadas aborda fenómenos tales como innovaciones evolutivas, heterocronías, constraints, entre otros, es decir, diversos procesos del desarrollo vinculados con cambios macroevolutivos. Así, a diferencia del programa anteriormente descrito, éste se centra en el análisis de cambios morfológicos entre especies, incluyendo el estudio de procesos del desarrollo a nivel celular y tisular, e incorporando el análisis de fósiles (además de la utilización de herramientas moleculares), entre otros elementos (Mabee 2000; Wu et al. 2006; Tokita et al. 2007; Müller 2008).

Para el análisis de las características temporales nos centraremos en uno de los principales temas de este programa de investigación: los constraints (restricciones). En principio pueden diferenciarse tanto restricciones del desarrollo como filéticas. ${ }^{7} \mathrm{Si}$ bien la definición de ambos tipos de restricciones continúa siendo debatida (ver por ejemplo Beldade y Brakefield 2003), se considera, en términos generales, que las restricciones del desarrollo son diversos factores internos de los sistemas de desarrollo que generan sesgos en la producción de variantes fenotípicas, o limitan la variabilidad fenotípica que puede darse en cada especie (Maynard Smith et al. 1985). Por otra parte, las restricciones filéticas surgen de la historia evolutiva previa de los organismos y canalizan, en mayor o menor medida, los cambios posibles posteriormente. Cabe destacar que la mayor parte de los autores no considera a las restricciones como meras limitaciones, dado que contribuyen al direccionamiento o canalización de los procesos evolutivos y del desarrollo (en particular, Arthur 2001 y Gould 2002). Analizaremos a continuación un caso clásico de restricción abordado desde la evo-devo que involucra al número de segmentos corporales de diferentes especies de ciempiés. El número de segmentos en esta clase de organismos presenta una gran variación, pero exhibiendo siempre un número impar. A partir del estudio del desarrollo ontogenético se encontró un mecanismo, basado en la expresión de genes de segmentación en dos etapas durante el desarrollo, que permite explicar esta constante: después de una primera etapa de expresión de genes de segmentación que determinan la separación de segmentos dobles, se expresan genes de segmentación en bandas intercaladas entre aquellos segmentos (y posteriormente se produce la unión de un segmento a la zona cefálica; el mecanismo detallado puede encontrarse en Chipman et al. 2004)..$^{8}$ Encontramos aquí, al considerar los aspectos ontogenéticos, características temporales similares a las ya analizadas en la sección 3.3, destacándose en particular la irreversibilidad: estos mecanismos generan regularidades muy resistentes al cambio, canalizándose así el desarrollo por determinados caminos y no otros. A su vez, tal irreversibilidad contribuye a explicar un fenómeno macroevolutivo: la persistencia evolutiva de una característica del plan corporal de esta clase de organismos a través de una gran extensión temporal.

\footnotetext{
${ }^{7}$ En cierto sentido las restricciones del desarrollo pueden considerarse un caso particular de las filéticas ya que también son producto de la historia evolutiva de cada especie (Gould y Lewontin 1979).

${ }^{8}$ Newman y Bath (2009) presentan otra interesante propuesta referente a la manera en que los procesos morfogenéticos y las redes de regulación génica asociadas a los mismos contribuyen a explicar la persistencia de determinadas características de los sistemas de desarrollo.
} 
Más allá de este vínculo entre estos procesos ontogenéticos y macroevolutivos, no resulta posible profundizar en este caso en el análisis de otras características temporales de los aspectos evolutivos dado que no se aborda en estos trabajos la historia evolutiva del grupo. Asimismo, nuestra taxonomía de características temporales parece más adecuada para analizar cambios en los sistemas biológicos más que fenómenos como este tipo de restricciones que implican la persistencia sin cambio de una característica propia de tales sistemas.

Para finalizar mencionaremos brevemente otro caso en el cual se aprecia la conjunción de los tiempos propios de los procesos del desarrollo y macroevolutivos: el estudio de las heterocronías, esto es, de los cambios evolutivos en la velocidad o momento de un proceso del desarrollo (Klingenberg 1998). Este tema ha sido fundamental en la historia de la evo-devo, siendo abordado por autores tales como Gavin De Beer, Pere Alberch, Gould, entre otros. Un ejemplo de estudio más actual de heterocronías corresponde al de la señal molecular $S h b$ en el desarrollo de dígitos del género Hemiergis (lagartijas) (Shapiro et al. 2003). En este trabajo se encontró que la duración de la expresión de Shb durante el desarrollo de diferentes especies de lagartijas correlaciona con el desarrollo de distinto número de dígitos en cada una de ellas. Se propuso entonces que este mecanismo heterocrónico estaría implicado en la evolución de las diferentes morfologías presentadas en este género. Así, encontramos involucradas en este ejemplo las características temporales propias de los procesos del desarrollo con la linealidad, no uniformidad e irreversibilidad propias de los procesos macroevolutivos. En conclusión, el análisis de estos ejemplos de restricciones y heterocronías muestra someramente de qué manera en este programa se vinculan los procesos del desarrollo que se repiten en cada generación con los cambios macroevolutivos.

\subsection{Eco-evo-devo}

En los últimos años, después de la consolidación de la evo-devo, comenzaron a surgir diversas propuestas de incorporación de temas y nuevos enfoques en el estudio de la evolución. Actualmente puede afirmarse que se ha generalizado el acuerdo acerca de 'ampliar' o extender la SM (Pigliucci y Müller 2010). En este contexto se origina una nueva sub-área dentro de la evo-devo, la denominada eco-evo-devo, en la cual se propone integrar, además de los procesos de desarrollo, las relaciones de los organismos con su ambiente en el estudio de la evolución (Gilbert y Epel 2009; Abouheif et al 2014). Uno de los principales temas estudiados desde la eco-evo-devo corresponde a los denominados 'procesos de inducción ambiental' (es decir, de generación de variantes fenotípicas evolutivamente relevantes debido a algún estímulo ambiental). El ambiente aparece así con una nueva función, diferente a la que presentaba en los procesos selectivos.

Asimismo, en eco-evo-devo se han diferenciado varios tipos de procesos que involucran la inducción ambiental y la plasticidad del desarrollo. Generalmente se distinguen los procesos de asimilación genética, acomodación genética y acomodación fenotípica (West-Eberhard 2003; Gilbert y Epel 2009). En primer lugar, en los procesos de asimilación genética (anteriormente estudiados por Conrad Waddington e Ivan Schmalhausen, figuras fundamentales para la historia de la eco-evo-devo, entre otros 
autores) se origina un nuevo fenotipo durante el desarrollo debido a la inducción ambiental y la plasticidad. Si el fenotipo así generado es adaptativo, la inducción persiste. Si a su vez existe la posibilidad de que ese cambio pueda surgir también por alguna variante genética, ésta será seleccionada, 'estabilizándose genéticamente', es decir, expresándose entonces el nuevo fenotipo independientemente del ambiente (Gilbert y Epel 2009).

Por otra parte, la acomodación genética corresponde a la estabilización de la plasticidad del desarrollo de una característica inducible por ambiente. Cabe esperar que esto suceda cuando una especie habita en condiciones ambientales diversas y no hay una variante que sea siempre más ventajosa que las otras. En este caso suele ser seleccionada la capacidad de expresión de diferentes variantes fenotípicas según se encuentre presente o no el factor ambiental inductor. Un ejemplo de acomodación genética cuyo mecanismo regulatorio ha sido dilucidado es el de la especie de polilla Manduca sexta (gusano del tabaco), la cual posee una larva con coloración marcadamente diferente al eclosionar en invierno (poseen color negro, lo cual aumenta la absorción de energía solar) o en verano (presenta coloración verde-turquesa que le proporciona mayor camuflaje y defensa frente a la predación) (Suzuki y Nijhout 2006).

Por último, la acomodación fenotípica corresponde al ajuste adaptativo mutuo entre diferentes aspectos de un fenotipo, siguiendo a algún cambio durante el desarrollo, sin modificación genética asociada (Pigliucci 2005; Gilbert y Epel 2009). Un elemento propio de los mecanismos del desarrollo que contribuye a explicar este fenómeno es el de las inducciones recíprocas. Este aspecto de la plasticidad fenotípica facilitaría la aparición de novedades morfológicas y es propuesto por algunos autores como un paso intermedio entre la aparición de fenotipos novedosos, su eventual asimilación genética, y el origen de adaptaciones (West-Eberhard 2003). Así, si bien la acomodación fenotípica sería netamente un proceso del desarrollo, se relacionaría también con procesos evolutivos, como ampliaremos más adelante.

En principio todos estos fenómenos y procesos parecen presentar una interesante combinación de diferentes características temporales. No haremos aquí un análisis pormenorizado de todas las categorías para cada uno de ellos, sino que nos limitaremos a señalar algunos puntos en donde se evidencia la combinación de características temporales propias de procesos del desarrollo, micro y macroevolutivos.

En términos generales los procesos de inducción ambiental son considerados irreversibles durante el ciclo de vida de los organismos, tal como hemos presentado en la sección 3.3. Pero, al mismo tiempo, se incorpora aquí cierta reversibilidad al ser contemplados en una escala temporal más extensa y considerarse también su dimensión evolutiva. Esta reversibilidad permite modelar estos procesos en forma similar a la que hemos descrito para los procesos microevolutivos de selección natural. Por ejemplo, los mencionados experimentos de acomodación genética realizados con el gusano del tabaco indican que es posible modificar las frecuencias de los fenotipos de coloración que hemos descrito (y llegar incluso a la fijación de uno de ellos en poblaciones experimentales) mediante selección artificial a través de la exposición a diferentes temperaturas durante el desarrollo (Suzuki y Nijhout 2006). Así, se asume también aquí la uniformidad propia de los procesos microevolutivos. Sin embargo, se introduce en los 
procesos de acomodación genética una novedad respecto del tipo de trayectoria ya que se admiten, como describimos, cambios cíclicos (estacionales) en las frecuencias de las diferentes variantes fenotípicas.

Otra novedad respecto del enfoque de la SM corresponde a cierta reversibilidad que se encuentra presente en la primera etapa de los procesos de asimilación, antes de que ocurra la estabilización genética de los fenotipos: si en esta etapa el ambiente cambia y el factor inductor deja de estar presente, los fenotipos inducidos desaparecerían de la población. La etapa posterior, después de la estabilización genética, corresponde ya a un típico proceso microevolutivo, en el cual la frecuencia poblacional de las variantes genéticas relacionadas con el fenotipo antes inducible variarán según las presiones de selección lo determinen (tal como ejemplificamos en la sección 3.1). Por lo tanto encontramos de allí en más, tanto la posibilidad de reversiones de los estados poblacionales de frecuencias génicas, como la uniformidad y linealidad (en términos de aumento de la aptitud media poblacional) propias de los procesos microevolutivos. Asimismo, hallamos otra diferencia importante respecto del enfoque de la SM referente a la duración tanto de los procesos de asimilación como de los de acomodación genética: dado que la inducción ambiental puede actuar sobre varios organismos al mismo tiempo, y que el ambiente inductor suele ser además el ambiente que selecciona esos fenotipos, es esperable que el 'tiempo de prueba' de los fenotipos y de cambio de frecuencias en las poblaciones sea más corto que en los modelos de la SM (Shimada et al. 2010). Así, al incorporar nuevos tipos de respuestas posibles de los organismos y las poblaciones a los cambios ambientales, la eco-evo-devo presenta un aspecto más 'flexible' del estudio de los procesos microevolutivos respecto del enfoque propio de la SM, en relación tanto con la trayectoria temporal como con la posibilidad de reversión y la duración de estos procesos.

Por último, como adelantamos, desde la eco-evo-devo se ha destacado el rol que todos estos procesos pueden tener en el origen de innovaciones evolutivas y de nuevas especies, es decir, en procesos macroevolutivos. Por ejemplo, West-Eberhard ha propuesto un modelo que combina todos estos procesos y sus diferentes características temporales en la explicación del surgimiento de nuevos fenotipos (West-Eberhard 2003; 2005; Gilbert y Epel 2009). En este modelo los nuevos fenotipos pueden surgir, tanto por mutaciones como por inducción ambiental. La acomodación o 'reorganización fenotípica' (West-Eberhard 2005) permite la generación de fenotipos viables como respuesta a ese primer cambio en el desarrollo. Así se genera una 'población de nuevas variables fenotípicas, que proveen material para la selección' (West-Eberhard 2005,2544 ), dando inicio a un típico proceso microevolutivo de selección natural (con la diferencia de que si el origen de la variante fue la inducción ambiental, es posible que los cambios poblacionales sean más rápidos). Posteriormente, si el nuevo fenotipo es adaptativo y tiene un componente genético, puede seguir la acomodación (o la asimilación) genética. De este modo se generarán cambios en las frecuencias génicas de la población, tal como ocurre en los procesos de selección modelados desde la SM.

De esta manera, las propuestas de eco-evo-devo integran los procesos del desarrollo ontogenético con los micro y macroevolutivos, recuperando la irreversibilidad y recursividad de los primeros, la reversibilidad y linealidad propias de 
los procesos selectivos microevolutivos, y vinculando ambos tipos de procesos en la explicación de la macroevolución, histórica e irreversible. La integración de todos estos procesos se ve facilitada porque los mecanismos propuestos permiten acortar la duración de los rangos temporales necesarios para la ocurrencia de fenómenos macroevolutivos, volviéndolos así abarcables y abordables con las metodologías y modelos utilizados para el estudio de la microevolución y del desarrollo ontogenético de los organismos.

\section{Recapitulación y conclusiones}

Para concluir el recorrido realizado resumiremos y analizaremos los principales resultados obtenidos. En la Tabla 1 presentamos las características generales de los procesos involucrados en la biología evolutiva (micro y macroevolutivos) y en la biología del desarrollo. En esta tabla destacamos $\left(^{*}\right)$ las principales diferencias encontradas entre los tres enfoques: la variabilidad de la tasa de cambio y la gran duración de los procesos macroevolutivos, la reversibilidad de los procesos microevolutivos, y la trayectoria helicoidal de los procesos genético-moleculares involucrados en el desarrollo. Además, con respecto a las características indicadas como coincidentes entre los enfoques, encontramos en algunos casos diferentes matices, los cuales hemos detallado a lo largo del trabajo (tales como la mencionada diferencia entre la linealidad entendida como aumento de la aptitud en la SM y la noción propia de la extensión macroevolutiva ligada a la naturaleza histórico-narrativa de esos procesos).

Tabla 1: Resumen de las características temporales propias de los procesos estudiados en la SM, la extensión macroevolutiva y la biología del desarrollo. Se destacan las principales diferencias encontradas entre los tres enfoques (*).

\begin{tabular}{|l|l|l|l|}
\hline $\begin{array}{l}\text { Tipo de proceso/ } \\
\text { Característica } \\
\text { temporal }\end{array}$ & $\begin{array}{l}\text { Procesos } \\
\text { microevolutivos }\end{array}$ & $\begin{array}{l}\text { Procesos } \\
\text { macroevolutivos }\end{array}$ & $\begin{array}{l}\text { Procesos } \\
\text { ontogenéticos }\end{array}$ \\
\hline Tipo de trayectoria & Lineal & Lineal & $\begin{array}{l}\text { - Lineal (enfoque } \\
\text { anatómico) } \\
\text { - Helicoidal (nivel } \\
\text { genético-molecular)* }\end{array}$ \\
\hline Tasa de cambio & Uniforme & Variable* & Variable \\
\hline Reversibilidad & Sí* & No & No \\
\hline Duración & $\begin{array}{l}\text { Larga (algunas } \\
\text { generaciones) }\end{array}$ & $\begin{array}{l}\text { Muy larga (miles de } \\
\text { generaciones)* }\end{array}$ & $\begin{array}{l}\text { Corta (una generación, } \\
\text { tiempo de vida individual) }\end{array}$ \\
\hline
\end{tabular}


A su vez, en la Tabla 2 resumimos las principales características encontradas en los dos principales programas de evo-devo y en eco-evo-devo. Se indica además cuáles de esas características son comunes con las encontradas en los enfoques previos de los fenómenos micro y macroevolutivos y cuáles son novedosas respecto de tales enfoques.

Tabla 2. Características generales de los procesos abordados por los principales programas de investigación de la evo-devo (genética evolutiva del desarrollo y embriología y morfología comparadas) y eco-evo-devo, considerando solamente su dimensión evolutiva. Destacado (*): características en común con la extensión macroevolutiva. Destacado (†): características en común con la SM. Sin indicación: características novedosas respecto de los otros enfoques de la evolución.

\begin{tabular}{|l|l|l|l|}
\hline $\begin{array}{l}\text { Campo de } \\
\text { estudio/ } \\
\text { Característica } \\
\text { temporal }\end{array}$ & $\begin{array}{l}\text { Genética } \\
\text { evolutiva del } \\
\text { desarrollo }\end{array}$ & $\begin{array}{l}\text { Morfología y } \\
\text { embriología } \\
\text { comparadas }\end{array}$ & Eco-evo-devo \\
\hline $\begin{array}{l}\text { Tipo de } \\
\text { trayectoria }\end{array}$ & Lineal* & Lineal* & $\begin{array}{l}\text { - Linealキ } \\
\text { - Cíclico (acomodación } \\
\text { genética) }\end{array}$ \\
\hline $\begin{array}{l}\text { Tasa de } \\
\text { cambio }\end{array}$ & Variable* & Variable* & $\begin{array}{l}\text { Uniforme (propia de los } \\
\text { procesos microevolutivos } \\
\text { pero explica discontinuidades) }\end{array}$ \\
\hline Reversibilidad & Sí & $\begin{array}{l}\text { Sí (propia de la SMキ + propia } \\
\text { de la inducción ambiental) }\end{array}$ \\
\hline Duración & $\begin{array}{l}\text { Muy larga } \\
\text { (macroevolutiva)* }\end{array}$ & $\begin{array}{l}\text { Muy larga } \\
\text { (macroevolutiva)* }\end{array}$ & $\begin{array}{l}\text { Larga } \text {, aún para los cambios } \\
\text { macroevolutivos }\end{array}$ \\
\hline
\end{tabular}

Veamos en primer lugar qué sugieren estos resultados acerca de la integración entre microevolución y macroevolución en los principales programas de investigación de la evo-devo. En principio, encontramos en ambos programas varias características en común con las propias de la extensión macroevolutiva de la SM. La tasa de cambio variable, la linealidad de los procesos y su extensa duración dan cuenta del importante rol de la macroevolución en evo-devo, ya que aporta el campo de fenómenos a ser explicado. En particular en el programa de la morfología y embriología comparadas se recuperan las cuatro características temporales propias de tales fenómenos. Sin embargo, reconocemos en el programa de la genética evolutiva del desarrollo dos diferencias importantes respecto del enfoque de la extensión macroevolutiva. Por una parte, la linealidad propia del enfoque histórico de la macroevolución se aplica en este programa a la evolución de entidades genético-moleculares. Además no encontramos aquí la irreversibilidad, una de las características fundamentales de la macroevolución, sino la reversibilidad propia de la metodología utilizada para el estudio de los procesos 
genético-moleculares del desarrollo. En este sentido la utilización de elementos explicativos del ámbito genético-molecular para dar cuenta de los fenómenos macroevolutivos evidencia dos supuestos de orden metodológico y epistemológico presentes en la genética evolutiva del desarrollo: cierta continuidad entre el ámbito genéticomolecular y el organísmico en el estudio del desarrollo ontogenético (presente también, como desarrollamos, en la biología del desarrollo) y, a su vez, un continuum entre los cambios organísmicos y los fenómenos macroevolutivos que se busca explicar. Asimismo, el primero de esos supuestos no se encontraría presente en el programa de la embriología y morfología comparadas, ya que en éste se incorporan más niveles de organización entre el genético-molecular y el organísmico para estudiar los mecanismos del desarrollo relacionados con procesos evolutivos. Con respecto al segundo supuesto sí podría vislumbrarse en este programa cierta continuidad entre los procesos organísmicos y los macroevolutivos. Como surge del análisis realizado, parece encontrarse en el programa de la morfología y embriología comparadas cierta relación directa entre los procesos del desarrollo de los organismos y los fenómenos macroevolutivos, los cuales se explicarían por la persistencia de esos mecanismos de desarrollo generación tras generación, a lo largo de una gran extensión temporal (macroevolutiva).

Por otra parte, en relación con las características temporales presentes en eco-evodevo, encontramos que una de las principales diferencias respecto de los dos programas de evo-devo analizados corresponde a la uniformidad de la tasa de cambio asumida en aquel campo. Este elemento resulta llamativo dada su importancia en las críticas de la extensión macroevolutiva a la SM. Así, aunque en ambos enfoques identificamos un rol claro de la macroevolución como presentador fenoménico, las características generales asumidas para los procesos no son coincidentes. A su vez, estas diferencias en el enfoque presentado por cada una de estas propuestas se relaciona con la recuperación en eco-evo-devo de varias características temporales propias de la SM: además de la uniformidad, se asume una menor duración requerida para los cambios macroevolutivos, así como la reversibilidad y linealidad de los procesos estudiados. De esta manera, aun cuando en eco-evo-devo se introducen elementos novedosos respecto de los estudios previos de la evolución, también se recuperan varias características propias de la SM y de los modelos de la genética de poblaciones. Estas características parecen evidenciar la reproducción en eco-evo-devo de uno de los principales supuestos de la SM: la continuidad entre los cambios microevolutivos, que ocurren a escala poblacional, y los fenómenos macroevolutivos. Así, la estrategia que propone la eco-evo-devo para la integración entre el desarrollo ontogenético, la macroevolución y la microevolución involucra la recuperación de varios elementos propios de la SM, incluyendo la relación de continuidad entre micro y macroevolución (además de los elementos novedosos resumidos en la Tabla 2).

En conclusión, el análisis de las características temporales recuperadas en estas propuestas de extensión de la SM ha brindado elementos para investigar en qué medida y de qué manera se integran en ellas enfoques previos de la evolución y del desarrollo ontogenético de los organismos. Esta indagación nos permitió dar cuenta de la recuperación de diferentes elementos propios de cada uno de esos enfoques, así 
como de algunas de las novedades presentes en estas propuestas integradoras y de los supuestos involucrados en tal integración. En términos generales, hemos reconocido la estrategia de asumir cierta continuidad entre el ámbito organísmico y el macroevolutivo en los dos principales programas de evo-devo (y también la continuidad entre los cambios organísmicos y los genético-moleculares en el programa de la genética evolutiva del desarrollo), y entre los ámbitos micro y macroevolutivo en eco-evo-devo. De esta manera, el análisis realizado ha permitido profundizar en la propia naturaleza subdisciplinar de la evo-devo, así como caracterizar el tipo de integración entre los aspectos microevolutivos, macroevolutivos y ontogenéticos en este campo de estudio. Por último, el presente trabajo muestra que la indagación de los cambios conceptuales involucrados en la extensión de la SM constituye un camino posible para el análisis crítico de las novedades que efectivamente comprenden estas prometedoras propuestas.

\section{REFERENCIAS}

Abouheif, E., M. J. Favé, A. S. Ibarrarán-Viniegra, M. P. Lesoway, A. M. Rafiqi, and R. Rajakumar. 2014.Eco-Evo-Devo: The time has come Adv. Exp. Med. Biol. 781: 107-125.

Alberch, P., S. J. Gould, G. F. Oster, y D. B Wake. 1979. Size and shape in ontogeny and phylogeny. Paleobiology 5(3): 296-317.

Amundson, R. 2005. The Changing Role of the Embryo in Evolutionary Thought Roots of Evo-Devo. Cambridge: Cambridge University Press.

Arthur, W. 2001. Developmental drive: an important determinant of the direction of phenotypic evolution. Evolution \& development 3(4): 271-278.

Basch, M. L., M. Bronner-Fraser y M. I. García-Castro. 2006. Specification of the neural crest occurs during gastrulation and requires Pax7. Nature 441(7090): 218-222.

Beldade, P. y P. M. Brakefield. 2003. The difficulty of agreeing about constraints. Evolution \& development 5(2): 119-120.

Chipman, A. D., W. Arthur y M. Akam. 2004. A double segment periodicity underlies segment generation in centipede development. Current Biology 14(14): 1250-1255.

Cohn, M. J. y C. Tickle. 1999. Developmental basis of limblessness and axial patterning in snakes. Nature 399(6735): 474-479.

Cracraft, J. 2005. Phylogeny and evo-devo: Characters, homology, and the historical analysis of the evolution of development. Zoology 108: 345-356.

Denver, R. J. 1998. Hormonal Correlates of Environmentally Induced Metamorphosis in the Western Spadefoot Toad, Scaphiopus hammondii. General and comparative endocrinology 110(3): 326-336.

Eldredge, N. y S. J. Gould. 1972. Punctuated equilibria: an alternative to phyletic gradualism. En Models in paleobiology, ed. T.J.M. Schopf, 82-115. San Francisco: Freeman, Cooper and Company.

Fox Keller, E. 2000. Lenguaje y Vida. Buenos Aires: Ediciones Manantial.

Gilbert S.F. y D. Epel. 2009. Ecological Developmental Biology: Integrating Epigenetics, Medicine, and Evolution. Sunderland, MA: Sinauer Associates Inc. Publishers.

Gould, S. J. 1970. Dollo on Dollo's law: irreversibility and the status of evolutionary laws. Journal of the History of Biology 3(2):189-212.

- 1977. Ontogeny and Phylogeny. Cambridge, Massachusetts: The Belknap Press of Harvard University press.

- 1987. Time's Arrow, Time's Cycle: Myth and Metaphor in the Discovery of Geological Time. Cambridge, Mass: Harvard University Press.

- 2002. The Structure of the Evolutionary Theory. Cambridge, Massachusetts: The Belknap Press of Harvard University Press.

Gould, S. J. y N. Eldredge. 1977. Punctuated equilibria: The Tempo and Mode of Evolution reconsidered. Paleobiology 3: 115-151. 
Gould, S. J. y R. C. Lewontin. 1979. The spandrels of San Marco and the Panglossian paradigm: a critique of the adaptationist programme. Proceedings of the Royal Society of London. Series B. Biological Sciences 205(1161): 581-598.

Hall, B. K. 1984. Developmental processes underlying heterochrony as an evolutionary mechanism. Canadian Journal of Zoology 62(1): 1-7.

Hendrikse, J. L., T. E. Parsons y B. Hallgrimsson. 2007. Evolvability as the proper focus of evolutionary developmental biology. Evolution \& development 9(4): 393-401.

Kempermann, G., H. G. Kuhn, y F. H. Gage. 1997. More hippocampal neurons in adult mice living in an enriched environment. Nature 386(6624): 493-495.

Klingenberg, C. P. 1998. Heterochrony and allometry: the analysis of evolutionary change in ontogeny. Biological Reviews 73(1): 79-123.

Krain, L. P. y R. J. Denver. 2004. Developmental expression and hormonal regulation of glucocorticoid and thyroid hormone receptors during metamorphosis in Xenopus laevis. Journal of Endocrinology 181(1): 91-104.

Laubichler M. D. y J. Maienschein. 2007. From embryology to Evo-devo: a history of developmental evolution. Cambridge, Massachusetts: The MIT Press.

Lee, P. N., P. Callaerts, H. G. de Couet y M. Q. Martindale. 2003. Cephalopod Hox genes and the origin of morphological novelties. Nature 424(6952): 1061-1065.

Love, A. C. 2006. Evolutionary morphology and Evo-devo: Hierarchy and novelty. Theory in biosciences 124: 317-333

Mabee, P. M. 2000. Developmental data and phylogenetic systematics: evolution of the vertebrate limb. American Zoologist 40(5): 789-800.

Maynard Smith, J., R. Burian, S. Kauffman, P. Alberch, J. Campbell, B. Goodwin, (...) y L. Wolpert. 1985. Developmental constraints and evolution: a perspective from the Mountain Lake conference on development and evolution. Quarterly Review of Biology. 265-287.

Mayr, E. 1942. Systematics and the Origin of Species. Nueva York: Columbia University.

Morin, E. 1983. El Método. vol. II: La vida de la vida. Madrid: Cátedra.

Müller, G. B. 2008. Evo-devo as a discipline. En Evolving Pathways: key themes in evolutionary developmental biology. A. Minelli y G. Fusco eds, 5-30. Cambridge: Cambridge University Press.

Newman, S.A y R. Bath. 2009. Dynamical patterning modules: a 'pattern language' for development and evolution of multicellular form. The International journal of developmental biology 53: 693-705.

Pick L. y A. Heffer. 2012. Hox gene evolution: multiple mechanisms contributing to evolutionary novelties. Ann N Y Acad Sci. 1256: 15-32.

Pigliucci, M. 2005. Evolution of phenotypic plasticity: where are we going now?. Trends in Ecology \& Evolution 20(9): 481-486.

Pigliucci, M. y G. Müller. 2010. Evolution - the extended synthesis. Cambridge, Massachusetts: The MIT Press.

Raff, R. A. y G. A. Wray. 1989. Heterochrony: developmental mechanisms and evolutionary results. Journal of Evolutionary Biology 2(6): 409-434.

Rasskin-Gutman, D y B. Esteve-Altava. 2009. Modeling Evo-Devo: Broken Hierarchies and Multiple Scales of Organization and Complexity. AIP Conference Proceedings 1167: 43-55.

Rendón, C. y G. Folguera. 2011. Jerarquías en biología: el vínculo entre la evolución y el desarrollo de los organismos, en Epistemología e historia de la ciencia: selección de trabajos de las XXI Jornadas, Vol. 17. N. Venturelli y M. Velasco eds. 371-379. Córdoba: Universidad Nacional de Córdoba.

Rendón C., G. Folguera y A. Massarini. 2012. Complejidad e interdisciplinariedad en el paisaje diversificado de la biología evolutiva del desarrollo (evo-devo). En Viaje a la Complejidad. N. Caparrós y R. Cruz Roche eds. (2): 178-192. Mexico y Buenos Aires: Biblioteca Nueva y Siglo XXI.

Rendón, C. 2013. Evo-devo como abordaje complejo de la evolución biológica. Ludus Vitalis, 21(39): 2742.

Ridley, M.R. 2004. Evolution (3ra edición). Cambridge, MA: Bllackwell Publishing.

Robert, J. S. 2002. How developmental is evolutionary developmental biology?. Biology and Philosophy 17: 591-611.

Ronshaugen, M., N. McGinnis y W. McGinnis. 2002. Hox protein mutation and macroevolution of the insect body plan. Nature 415(6874): 914-917. 
Seilacher, A. 1984. Constructional morphology of bivalves: evolutionary pathways in primary versus secondary soft-bottom dwellers. Palaeontolog, 27(2): 207-237.

Shapiro, M. D., J. Hanken y N. Rosenthal. 2003. Developmental basis of evolutionary digit loss in the Australian lizard Hemiergis. Journal of Experimental Zoology Part B: Molecular and Developmental Evolution 297(1): 48-56.

Shimada, M., Y. Ishii y H. Shibao. 2010. Rapid adaptation: a new dimension for evolutionary perspectives in ecology. Population ecology 52(1): 5-14.

Simpson, G. G. 1953. The Major Features of Evolution. New York: Columbia University Press.

Smith, K. K. 2003. Time's arrow: heterochrony and the evolution of development. International Journal of Developmental Biology 47 (7/8): 613-622.

Suzuki, Y. y H. F. Nijhout. 2006. Evolution of a polyphenism by genetic accommodation. Science 311(5761): 650-652.

Tokita, M., T. Kiyoshi y K. N. Armstrong. 2007. Evolution of craniofacial novelty in parrots through developmental modularity and heterochrony. Evolution \& development 9(6): 590-601.

Wake, D. B., G. Roth y M. H. Wake. 1983. On the problem of stasis in organismal evolution. Journal of Theoretical Biology 101(2), 211-224.

West-Eberhard, M. J. 2003. Developmental plasticity and evolution. New York: Oxford University Press.

- 2005. Developmental plasticity and the origin of species differences. Proceedings of the National Academy of Sciences of the United States of America 102(Suppl 1): 6543-6549.

Wu, P., T.X. Jiang, J.Y. Shen, R.B. Widelitz y C.M. Chuong. 2006. Morphoregulation of avian beaks: comparative mapping of growth zone activities and morphological evolution. Developmental Dynamics 235(5): 1400-1412.

Zúñiga, A., A. P. G. Haramis, A.P. McMahon y R. Zeller. 1999. Signal relay by BMP antagonism controls the $\mathrm{SHH} / \mathrm{FGF} 4$ feedback loop in vertebrate limb buds. Nature 401(6753): 598-602.

Constanza Alexandra Rendón es Licenciada en Ciencias Biológicas por la Facultad de Ciencias Exactas y Naturales (FCEN) de la Universidad de Buenos Aires (UBA). Actualmente se desempeña como becaria de CONICET y se encuentra terminando su tesis doctoral en la temática de Filosofía de la Evo-devo en la facultad de Ciencias Exactas y Naturales (FCEN) de la Universidad de Buenos Aires (UBA).

Direccrón: Grupo de Filosofía de las Ciencias, Facultad de Ciencias Exactas y Naturales, Universidad de Buenos Aires, Intendente Güiraldes 2160, Ciudad Universitaria, Pabellón II, Ciudad Autónoma de Buenos Aires, C1428EGA, Argentina. E-mail: constanzarendon@yahoo.com

Guillermo Folguera es Doctor en Ciencias Biológicas por la Facultad de Ciencias Exactas y Naturales (FCEN) de la Universidad de Buenos Aires (UBA). En cuanto a su formación de grado es Licenciado en Filosofía de la Facultad de Filosofía y Letras (FFyL) y en Ciencias Biológicas de la FCEN, ambas carreras realizadas en la UBA. Actualmente se desempeña como investigador adjunto CONICET en la temática de Filosofía de la Ciencia. Es Profesor Adjunto de la disciplina Historia de la Ciencia de la FCEN. A su vez, dirige un proyecto de investigación en la UBA que aborda la el área de la Filosofía de la Biología. Por otro lado, ha realizado diversas estancias postdoctorales en el exterior a la vez que ha dictado cursos de postgrado en las temáticas de filosofía de la ciencia. Por último, es autor de dos libros y de numerosos artículos en revistas nacionales e internacionales.

Dirección: Grupo de Filosofía de las Ciencias, Facultad de Ciencias Exactas y Naturales, Universidad de Buenos Aires, Intendente Güiraldes 2160, Ciudad Universitaria, Pabellón II, Ciudad Autónoma de Buenos Aires, C1428EGA, Argentina. E-mail: guillefolguera@yahoo.com.ar 